\title{
Myasthenia Gravis: Two Case Reports and Review of the Literature
}

\author{
Ana Laura Colle Kauling ${ }^{1}$, Maria Cristina Simões de Almeida ${ }^{2}$, Giovani de Figueiredo Locks, TSA ${ }^{3}$, \\ Guilherme Muriano Brunharo 4
}

Summary: Kauling ALC, Almeida MCS, Locks GF, Brunharo GM - Myasthenia Gravis: Two Case Reports and Review of the Literature.

Background and objectives: Myasthenia gravis (MG) is an autoimmune neurologic disease that affects the postsynaptic portion of the neuromuscular junction. It represents a challenge for anesthesiologists due to the diversity of disease manifestations and possibility of postoperative respiratory complications. The objective of this study was to demonstrate the importance of adequate monitoring of the neuromuscular blockade (NMB) due to the multiple presentations of MG.

Contents: In this paper we report two cases of patients with MG. The first patient presented with the classical sensitivity to the neuromuscular blocker (NMB) and the second had a similar response to that of a normal patient. The literature review will be restricted to disease characteristics, while the description of its pathophysiology will focus on its reactions to NMB.

Conclusions: We suggest that, due to the multiple presentation and treatment of MG, neuromuscular transmission monitors are fundamental when using NMB.

Keywords: Neuromuscular Blocking Agents; Myasthenia gravis; Electromyography; Atracurium; Anesthesia, General; Monitoring, Physiologic.

\section{INTRODUCTION}

Myasthenia gravis (MG) is an autoimmune neurologic disease that affects the post-synaptic portion of the neuromuscular junction (NMJ). Over the last years, the understanding of the neuromuscular transmission (NMT) and nature of the disease provided better treatment with low mortality, making the expression MG almost unjustifiable ${ }^{1-5}$.

The precise origin of the immune response is unknown, but thymic abnormalities almost certainly play a relevant role in the genesis of anti-motor plate nicotinic receptor antibodies. These antibody reactions activate the complement system, resulting in damage to the muscular membrane and sodium channels with significant disruption of NMT ${ }^{2-8}$.

Despite advanced diagnostic and treatment techniques, MG still represents a challenge to anesthesiologists, justified by the several disease manifestations and the possibility

Received from Universidade Federal de Santa Catarina (UFSC), Brazil.

1. Resident Physician of the Anesthesiology Sector of the Hospital das Clínicas da Faculdade de Medicina da Universidade de São Paulo (FMUSP)

2. Medical degree from Johannes Gutenberg Universität Mainz, Germany; Professor of the

Departamento de Cirurgia da Universidade Federal de Santa Catarina (UFSC)

3. Anesthesiologist of Hospital Universitário da UFSC

4. Resident Physician of the Anesthesiology Sector of the Hospital Governador Celso Ramos

Submitted on September 29, 2010.

Approved on February 21, 2011.

Correspondence to:

Dra. Ana Laura Colle Kauling

Av. Dr. Enéas de Carvalho Aguiar, 155

05403000 - São Paulo, SP, Brazil

E-mail: analaurakauling@gmail.com of severe postoperative ventilatory complications ${ }^{9,10}$. In the present article we report two cases of MG patients who had different reactions to neuromuscular blockers (NMB), and the literature review will focus on aspects of the disease and its pathophysiology related to reactions to NMB.

\section{CASE REPORTS}

\section{Case $1^{11}$}

This is a 55-year old male patient, $82 \mathrm{~kg}, 167 \mathrm{~cm}$, with history of MG who was admitted to undergo transternal thymectomy. He reported chronic use of prednisone $60 \mathrm{mg}^{-d a y^{-1}}$, and

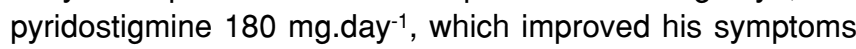
considerably. He had no other comorbidities. Laboratory tests and electrocardiogram were normal. Chest X-ray and tomography showed image compatible with increased thymic size.

The patient received no premedication and was referred to the operating room on the morning of the surgery without interrupting his daily medications. In the operating room, he was monitored with ECG on DII and V5, automatic noninvasive measurement of blood pressure, pulse oximetry, capnography, and electromyography of the adductor pollicis muscle.

After administration of $100 \%$ oxygen by face mask, he received propofol $150 \mathrm{mg}$, and alfentanil 1,500 $\mu \mathrm{g}$. After loss of the palpebral reflex, electromyography (Relaxograph ${ }^{\circledR}$ ) with stimulating electrodes placed on the wrist, on the trajectory of the ulnar nerve, was performed with supramaximal stimuli every 20 seconds with frequency of $2 \mathrm{~Hz}$ and train-of-four stimulus (TOF) (Figure 1). 


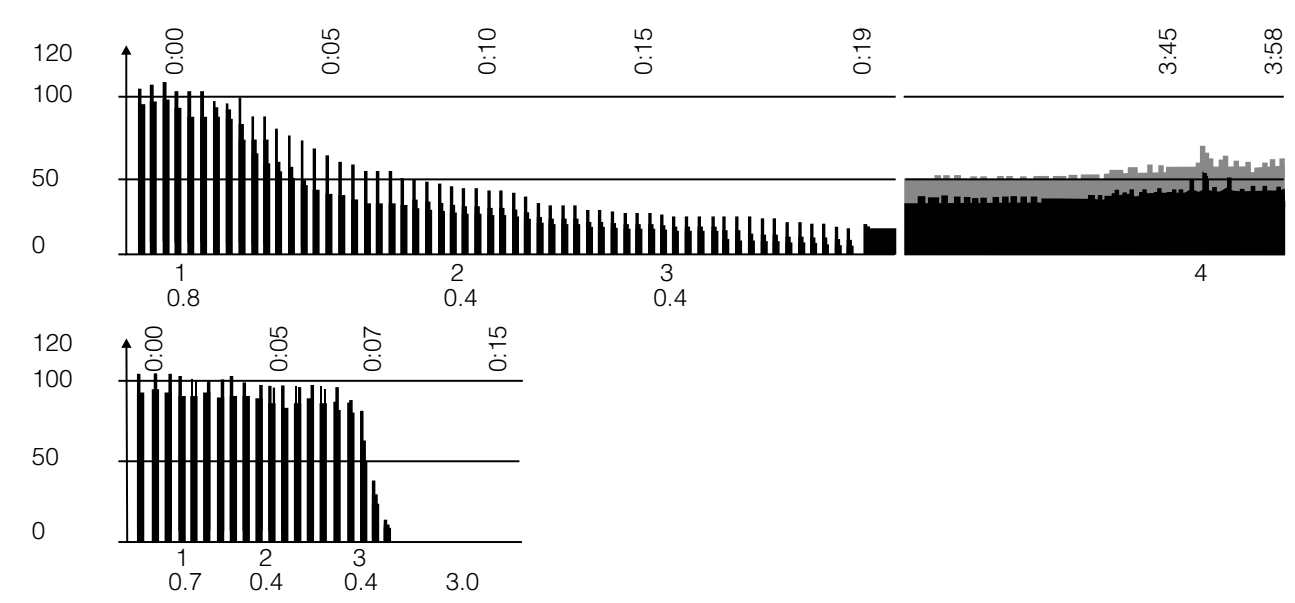

Figure 1 - Electromyographic Tracing of TOF of Adductor Pollicis Muscle in a Patient with MG who Received Fractionated Doses of Cisatracurium. The lower tracing shows the response of a normal patient to the same fractionated doses of cisatracurium ${ }^{11}$. With author's and Brazilian Society of Anesthesiology's permission.

After a short period of stabilization of the tracing, cisatracurium $0.8 \mathrm{mg}$ was administered with an expressive reduction in muscle contraction. Two additional doses of $0.4 \mathrm{mg}$ were administered before the patient was considered to have a satisfactory degree of muscle relaxation for tracheal intubation. Anesthesia was maintained with isoflurane, nitrous oxide, and fentanyl. At the end of the procedure, which lasted 3 hours and 45 minutes, the $\mathrm{T} 4 / \mathrm{T} 1$ ratio was 0.75 . We decide to attempt pharmacologic reversion with neostigmine $0.05 \mathrm{mg}^{\mathrm{kg}}{ }^{-1}$, which was considered unsatisfactory despite the patient had an excellent respiratory pattern. The patient was transferred to the Intensive Care Unit intubated and on assisted ventilation, where he remained for 2 hours with ventilatory assistance. At the end of this period, with decurarization patterns that were considered satisfactory $(\mathrm{T} 4 / \mathrm{T} 1>0.9)$, the patient was extubated. His evolution was satisfactory and he was discharged on the sixth postoperative day.

\section{Case 2}

This is a 55-year old female patient, $64 \mathrm{~kg}, 165 \mathrm{~cm}$, physical status ASA III who was admitted for a mastectomy for breast

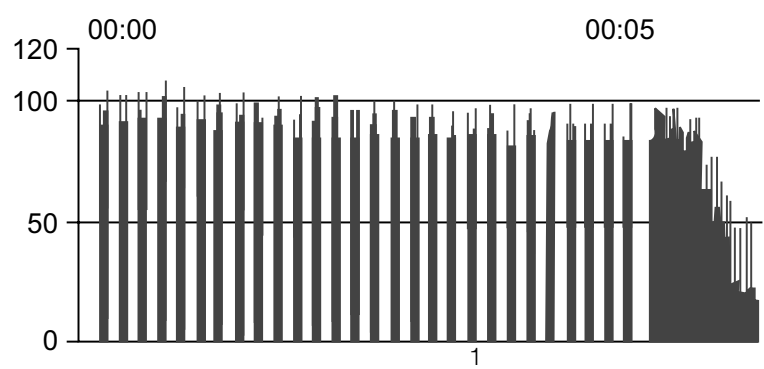

Figure 2 - Electromyographic Tracing of the Adductor Pollicis Muscle in a Patient with MG who Received Initially $3.5 \mathrm{mg}$ and, after 4 Minutes, $31.5 \mathrm{mg}$ of Atracurium. The initial tracing did not show muscle fatigue, and muscle relaxation was observed only with a dose of $0.5 \mathrm{mg} \cdot \mathrm{kg}^{-1}$ of atracurium. carcinoma. On preoperative evaluation, she reported history of diabetes mellitus and use of insulin, MG for five years and use of valproic acid, amitriptyline, and prednisone. Her laboratorial tests, electrocardiogram, and Chest X-ray were normal.

On the morning of the surgery the patient was transferred from the Intensive Care Unit, arriving at the operating room awake, oriented, dyspneic, with $\mathrm{SpO}_{2} 89 \%$, ventilating with oxygen by a face mask. Monitoring consisted of ECG on DII and $\mathrm{V} 5$, automatic noninvasive measurement of blood pressure, pulse oximetry, capnography, electromyography (Relaxograph ${ }^{\circledR}$ ), and accelerometry (TOF Watch ${ }^{\circledR}$ ) of adductor pollicis muscles, with one monitor on each arm.

After inhalation of $100 \%$ oxygen by a face mask for three minutes, fentanyl $200 \mu \mathrm{g}$ and propofol $200 \mathrm{mg}$ were administered. An initial dose of $3.5 \mathrm{mg}$ of atracurium, complemented to $0.5 \mathrm{mg}^{\mathrm{kg}}{ }^{-1}$ four minutes after the initial dose, was administered. Anesthesia was maintained with sevoflurane, oxygen, and $50 \%$ air.

After anesthetic induction, muscle contraction was verified by electromyography of the adductor pollicis muscle through stimulator electrodes placed on the wrist, on the trajectory of the ulnar nerve. Evoked contraction was performed with supramaximal stimuli every 20 seconds, frequency of $2 \mathrm{~Hz}$, with TOF stimulus (Figure 2). Simultaneously, indirect muscle strength was recorded with an acceleration transducer placed on the contralateral thumb. Both electromyography and accelerometry, performed before the administration of atracurium showed no muscular fatigue.

At the end of the procedure, the patient recovered the muscle strength spontaneously, being extubated with T4/T1 of 0.9. With the patient awake, ventilating with $\mathrm{O}_{2}$ via a face mask, her $\mathrm{SpO}_{2}$ was above $90 \%$.

\section{DISCUSSION}

Myasthenia gravis is a chronic autoimmune disease that usually manifests in young adults or in the elderly, being charac- 
terized by weakness and fatigue of skeletal muscles due to repetitive use $3,4,6,12-14$.

Myasthenia gravis geoepidemiology shows that it is a rare disorder with similar incidence and prevalence in the world, except for infantile MG, which is more common in Asia 15-17. The incidence has increased in the last decades, going from $2-5 / 1,000,000$ to $9-21 / 1,000,000$, but without proportional increase in mortality. The disease attacks mainly women in the third and fourth decades of life in the proportion of 3:2 ${ }^{15}$.

Environmental and microbial agents have been suggested for its etiology, and the association between the disease and hepatitis $C$ virus have been reported. Cross-reaction between antibodies of MG patients with herpes simplex virus, besides other virus, has been reported ${ }^{18-22}$. Genetic predisposition for the disease is equally important 23,24 .

Whether there are predisposing factors is not established, but in some cases the presence of infection, emotional stress, surgeries, trauma, use of antibiotics, or pregnancy have been related to the onset of disease manifestation 1.

Ocular and palpebral muscle involvement is at times the only manifestation of MG with symptoms of diplopia and palpebral ptosis. These muscles have particularities that should be mentioned: they are fatigue resistant, have high blood flow for motor units and high mitochondrial content, therefore, they have high metabolic rate. Motor neurons in this area are anatomically small with high discharge frequencies; some muscles have multiple innervations where the end-plate potential, more than the action potential itself, is directly responsible for muscle activation. This means that any reduction in endplate potential has direct repercussion on muscle contraction. The role of immature or fetal receptors on extraocular muscle compromise in MG is controversial, but a factor that makes them susceptible in this disease is without any doubt the low expression of complement system regulators, making them susceptible to muscle membrane damage by this system, which is activated by the antigen-antibody reaction ${ }^{25}$. Besides the compromise of palpebral muscles, the involvement of face and bulbar muscles can be incapacitating and life-threatening ${ }^{3}$. Table I shows the percentage of muscular involvement in MG.

Although many aspects of MG still have no convincing explanation, there is no doubt about the immunologic character of the disease, proven by the substantial improvement of patients with plasmapheresis ${ }^{26,27}$. Antibodies, usually IgG1 and $\lg G 3$, are capable of activating the complement system ${ }^{2}$. The nature of these immunoglobulins indicates that they are T lymphocyte-dependent and that thymic cells type ED4 help $\mathrm{B}$ cells in their production ${ }^{28,29}$. Thus, in an expressive percentage of patients, especially young ones, the thymus is abnormal ${ }^{4}$. Despite a significant number of patients with thymic involvement, the presence of other sites of formation of these antibodies has been suggested, since patients are clinically improved after thymectomy but not cured ${ }^{30}$.

The main focus of these antibodies is, without any doubt, the NMJ, site of many drug interactions and intoxications because in this region there is no hematologic barrier ${ }^{31,32}$. Thus, similar to MG, other autoimmune diseases that also interfere
Table I - Percentage of Muscle Involvement in MG 1,2,15

\begin{tabular}{ll}
\hline Muscles & Percentage of involvement \\
\hline Ocular & $\mathbf{1 7 \%}$ \\
Ocular and bulbar & $\mathbf{1 3 \%}$ \\
Mild/moderate & $2 \%$ \\
Moderate/severe & $11 \%$ \\
Ocular and limbs & $\mathbf{2 0 \%}$ \\
Generalized & $\mathbf{5 0 \%}$ \\
Mild & $2 \%$ \\
Moderate & $14 \%$ \\
Severe & $15 \%$ \\
Requiring ventilatory assistance & $11 \%$ \\
Death despite ventilatory assistance & $8 \%$ \\
\hline
\end{tabular}

MG: Myasthenia Gravis.

with muscle contraction have been identified. Among them we could mention the reaction against calcium channels in Lambert-Eaton myasthenic syndrome and against potassium channels in congenital neuromyotonia ${ }^{2}$.

Most patients have anti-muscular nicotinic receptor antibodies, and there are those being considered a special subgroup of MG. In such patients, antibodies against muscle-specific kinase, a molecule in the proximity of the muscular nicotinic receptor that maintains the anatomic integrity of the NMJ, are detected ${ }^{30}$. Note that antibodies in MG do not attack subunits $\alpha 3$ or $\alpha 4 \beta 2$ of the nicotinic receptors, explaining the absence of autonomic and central nervous system symptoms ${ }^{33}$. Finally, in $10 \%$ of patients, antibodies are not detected; however, they have satisfactory response to plasmapheresis, and injection of plasma from these patients induces $M G$ in animals, suggesting that, even though antibodies are not detected by traditional methods, there must be an antibody-producing mechanism involved in this type of MG $3,4,13$. Clinical evolution, age, involvement of human leukocyte antigen, antibodies against nicotinic, and ryanodine receptors, besides the presence of thymic disease, help classify and predict the evolution of the disease. Myasthenia gravis classification according to clinical and laboratory aspects is presented in Tables II and III, respectively.

To understand the pathophysiology of $M G$ and reaction to NMB it is important among other aspects to understand the ways of maintaining the anatomic integrity of NMJ and muscular nicotinic receptor function when occupied by the neurotransmitter. Didactically, two important mechanisms maintain neuromuscular junction trophism. The first is the electrical activity from the motor neuron that affects the entire muscular surface, and the second is the involvement of molecular signals equally from axonal origin ${ }^{30}$.

The normal electrical activity from the undivided nerve inhibits acetylcholine receptor formation in all muscle nuclei 
Table II - Classification of MG According to the Ossermann Scale ${ }^{34}$

\begin{tabular}{ll}
\hline Type I & Ocular myasthenia characterized by ptosis and diplopia \\
Type Ila & Slow onset, frequently ocular, with gradual evolution to skeletal musculature \\
Type IIb & Slow onset with dysarthria, dysphagia, and changes in mastication \\
Type III & Fast onset with severe bulbar and skeletal muscle fatigue with respiratory muscle compromise \\
Type IV & Severe MG that manifests in two years \\
\hline
\end{tabular}

MG: Myasthenia Gravis.

Table III - Classification of MG in Subgroups ${ }^{3,13,15}$

\begin{tabular}{|c|c|c|c|c|}
\hline Subgroups & $\begin{array}{l}\text { Age } \\
\text { (years) }\end{array}$ & HLA association & Thymic disease & Antibodies \\
\hline \multicolumn{5}{|l|}{ MG with anti-AchR antibody: } \\
\hline early manifestation & $<40$ & DR3B8 & Hyperplasia & AchR \\
\hline late manifestation & $>40$ & DR2B7 (weak) & Normal for age & AchR, ryanodine and titin receptors* \\
\hline \multirow[t]{2}{*}{ Thymoma } & Variable & Unknown & Tumor & AchR \\
\hline & & & & AchR, ryanodine and titin receptors ${ }^{*}$ \\
\hline Antibody with low AchR affinity & Variable & Unknown & $\begin{array}{l}\text { Some cases of } \\
\text { hyperplasia }\end{array}$ & Little affinity for AchR \\
\hline Ocular MG & Variable & Unknown & Unknown & AchR $50 \%$; AchR with low affinity \\
\hline MuKi-MG & Variable & DR14DQ5 & Normal & MuKi \\
\hline Anti-AchR/MuKi antibody negative & Variable & Unknown & Not clear & Negative \\
\hline E-LMS & $20-60$ & DR3B8 & No reported & VGCa ${ }^{++} R$ \\
\hline E-LMS scLC & $>40$ & Unknown & Not reported & VGCa ${ }^{++} \mathrm{R}$ \\
\hline Neuromyotonia & $20-60$ & Unknown & Maybe thymoma & $V K^{+} R$ in $40 \%$ \\
\hline
\end{tabular}

MG: Myasthenia Gravis. AchR: acetylcholine receptor. HLA: human leukocyte antigen. DR3B8, DR7B7, DR14DQ5: subtypes of human leukocyte antigen. *titin: giant filamentous muscular protein essential for muscular development and structure, and muscular function. MuKi: muscle-specific kinase. Ag: antigen. E-LMS : EatonLambert Myasthenic Syndrome. VGCa++R: voltage-gated calcium receptor. scLC: small-cell lung carcinoma. VGK+R: voltage-gated potassium receptor.

except in subsynaptic nuclei. The direct consequence, when there is normal nerve activity, is the reduction in extrajunctional receptor formation and stimulation of motor plate receptor formation. Two substances (agrin and neuregulin) mediated by muscle-specific kinase are involved in maintaining the trophism of the motor plate. They are nerve-derived and bound to the basement lamina ${ }^{35-37}$. Some other forms of agrin, such as in blood vessels, kidneys, and muscles similar to that found in the motor plate do not lead to the formation or aggregation of acetylcholine receptors in the NMJ ${ }^{30}$. An experimental study ${ }^{38}$ suggests that neuronal agrin regulates both the differentiation of the pre-synaptic region and the muscular subsynaptic region. This molecule works in the muscle subsynaptic nucleus and induces the expression of acetylcholine receptors and their aggregation on the surface of the muscle membrane in the proximity of the axon terminal. Equally important in this mechanism is the presence of rapsin. Muscle-specific kinase is also involved on kinase receptors and neuregulin, which also interfere in the formation of acetylcholine receptors and their expression on the muscle membrane and sodium receptors ${ }^{3,39-44}$. In MG, the presence of muscle-specific kinase antibodies changes these complex mechanisms that maintain trophism and, as a result, an impoverishment of junctional acetylcholine receptors and an increase in extrajunctional acetylcholine receptors is observed ${ }^{45}$, a mechanism similar to that observed in patients whose binomial nerve-muscle is interrupted ${ }^{30}$.

The neuromuscular junction is a complex synapse that has three distinct components: presynaptic axonal terminal, site of acetylcholine synthesis and storage; synaptic cleft; and postsynaptic membrane, where nicotinic receptors and acetylcholinesterase are located ${ }^{30}$. Normal NMT begins when a nerve action potential arrives to the axonal presynaptic terminal, generating a calcium influx that penetrates in the 
axon through specific type $P$ and $Q$ calcium channels, called voltage-gated. They are opened whenever there is change in membrane voltage ${ }^{43}$. Calcium penetrates in the axon and, by acting on calmodulin, it releases acetylcholine vesicles from the cellular cytoskeleton. These free-vesicles move and go to the axonal periphery in the pre-synaptic portion of the motor plate. Through mechanisms involving molecules bound to the axonal membrane the membrane vesicle and the axonal membrane merges leading to acetylcholine exocytosis, all calcium-dependent mechanisms. The basement membrane is also located in the synaptic cleft. Proteins such as collagen, laminin, fibronectin, and perlecan on this structure are important components for efficient NMT. ColQ, a molecule similar to collagen, connected to acetylcholinesterase, is the characteristic example of a basement membrane-bound substance fundamental in the NMT mechanism ${ }^{46}$.

Once released on the synaptic cleft acetylcholine molecules occupy muscular acetylcholine receptors besides other neuronal receptors, and in special situations the extrajunctional receptors. To increase the contact area the postsynaptic membrane forms invaginations into the interior of the muscular cell, where nicotinic receptors anchor and remain on their crests, while sodium channels are on their deeper portions ${ }^{43,47}$.
The key elements of the postsynaptic region are, without any doubt, the muscular acetylcholine receptor and calcium molecules. Once acetylcholine molecules are bound to $\alpha 1$ and $\varepsilon$ and $\alpha 1$ and subunits on the extracellular portion of the receptor, they cause a physiologic torsion of approximately 10 degrees, especially in $\alpha$ subunits, resulting in anatomical modification of the pore, located in the transmembrane portion. Through the central pore, now with greater diameter, sodium ions enter and initiate an action potential in the postsynaptic region changing membrane polarity also known as "end-plate potential" $36,44,48,49$. In normal adults this potential is much higher than necessary to generate an action potential in the muscle cell, and this was called the "NMT safety factor". The action of acetylcholinesterase nullifies the effects of acetylcholine 3,30,42. Figure 3 shows the physiologic sites that acetylcholine binds to the muscle nicotinic receptor and the movement of the receptor, resulting in the opening of the central pore.

Because 17 genes encode muscle cholinergic receptors scientists have great difficulties in determining the mutations of these receptors ${ }^{46}$. Thus, some functions are regulated by more than one gene, and different mutations may result in disease with the same phenotype. This is exemplified by at least 56 mutations that cause Congenital Myasthenic Syndromes,
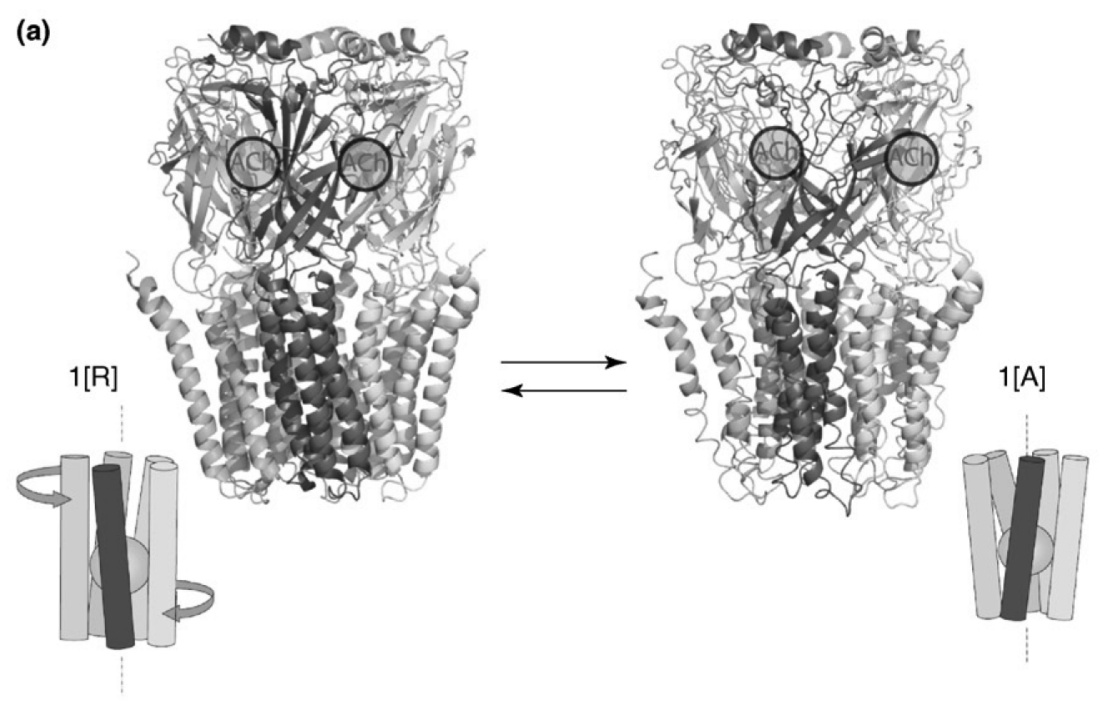

(b)
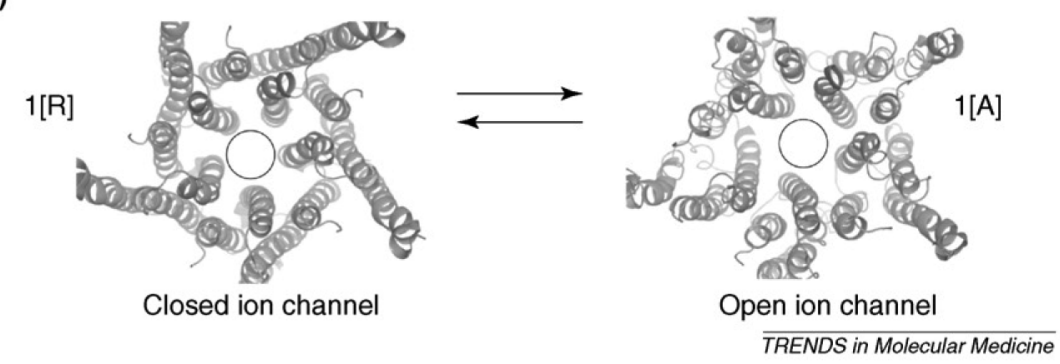

Figure 3 - Nicotinic Receptor: Allosteric Transition Model Known as "Twisted quaternary Model". In (a): lateral view of the resting and activated receptor model. A schematic representation of the moving quaternary structure is equally demonstrated as cylinder. In (b): transmembrane area of the resting and active models, similar to that demonstrated in (a). Reproduced with permission of Changeux JP ${ }^{44}$ and the editor. 
a group of diseases causing changes in NMT ${ }^{46}$. They were classified into presynaptic, synaptic, and postsynaptic. The first was described in children who had normal motor plate, but reduced size acetylcholine vesicles. This group comprises entities with decreased number of acetylcholine molecules released during exocytosis ${ }^{46}$. Synaptic disorders are related to acetylcholinesterase deficiency. This is a genetic defect of ColQ. On chronic deficiency due to genetic mutation of this molecule, more precisely on the 3p24.2 locus, there is a reduction in the bioavailability of acetylcholinesterase on the synaptic cleft, and consequently excess of acetylcholine. This leads to repetitive and persistent muscle stimulation resulting in desensitization of the motor plate nicotinic receptors. This is a rare disease with only 17 cases described in the literature ${ }^{50}$. Congenital Myasthenic Syndromes classified as postsynaptic are related to abnormalities in muscular nicotinic receptors ${ }^{46}$. The main mutations observed are located in $\alpha 1$, $\beta 1$, and $\varepsilon$ subunits ${ }^{46}$, so that the abnormal receptor does not respond with physiologic movement when acetylcholine molecules bind to it, and the central pore does not allow sodium molecules to go through ${ }^{44}$.

Based on the mutations observed in MG, three distinct receptor behaviors have been described. The first, called "function gain" in which mutations results in slow closure of the central pore and greater receptor affinity for acetylcholine. They are also known as "slow channel syndromes" 51. Neurotransmission is compromised by the excessive cation load that leads to the destruction of muscular invaginations of the motor plate, receptor desensitization, and motor blockade due to depolarization ${ }^{46}$. It is the most common type of the disease. The second is called "loss of function". In this situation the inverse mechanism is seen, i.e., the pore clos- es early. This type of MG is called "fast channel syndrome". The response to acetylcholine is greatly reduced despite the increased amount of acetylcholine quantum released by the axon. And in the third behavior there are situations in which both changes are present ${ }^{46}$. In all three dysfunctions a significant compromise of the NMT is observed ${ }^{43}$; there are different approaches and pharmacologic responses to treatment of $M G$ in these three modalities of receptor reaction. Figure 4 shows the graphic representation of subunit $\alpha 1$ mutations in MG.

In MG, besides channel dysfunction, the architecture of the muscle membrane is also compromised especially due to the complement system ${ }^{3,4,52}$. The immediate consequence is change in the quality and speed of the "end-plate potential" leading to dysfunction of the voltage-gated sodium channel. The final result is the loss of the "NMT safety factor" causing muscle fatigue and weakness ${ }^{5}$. A recent study suggested that besides changes in the motor plate there is also more distant compromise, most precisely in the actin-myosin system of muscle excitation/contraction. This is observed particularly in patients with the generalized form of the disease who usually have a thymoma, which may be another contributing factor for disease symptoms ${ }^{53}$.

Thymectomy is the characteristic situation in which the patients will undergo anesthesia. Care of MG patients is basically focused on the preoperative use of anticholinesterase drugs, reaction to NMB, and possible transoperative drug interactions ${ }^{54,55}$. Factors that can lead to the need of postoperative ventilatory assistance are equally important 56,57 . Thus it has been suggested that treatment with anticholinesterase drugs should not be interrupted before surgery, and one should prefer regional anesthesia whenever possible ${ }^{9}$. (a)

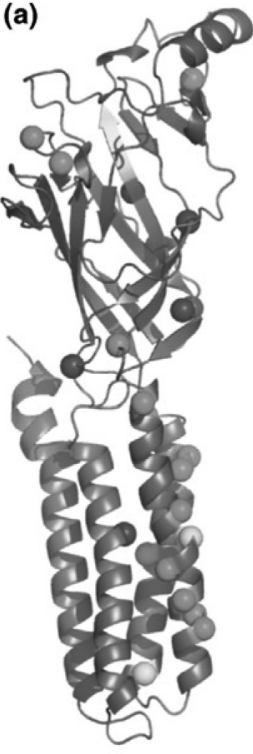

(b)
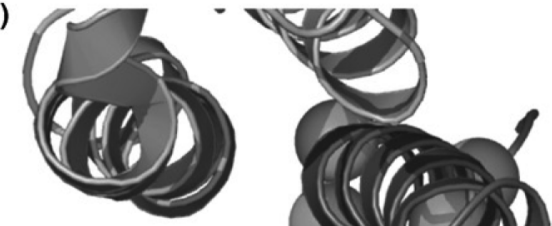

Gain of function

Loss of function
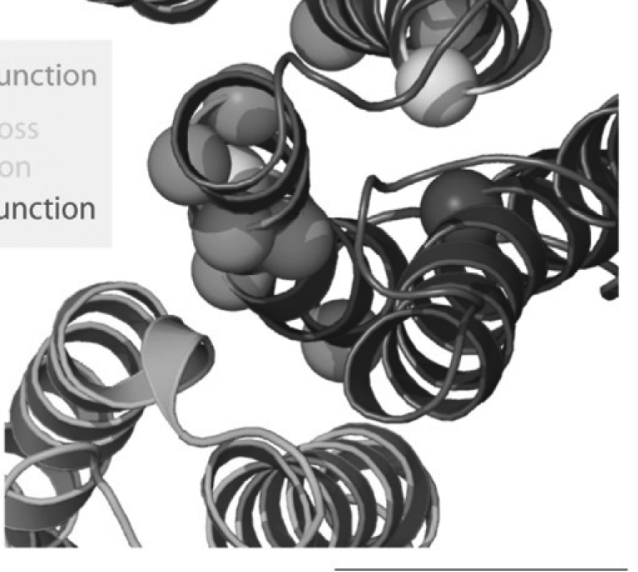

$\overline{\text { TRENDS in Molecular Medicine }}$

Figure 4 - Distribution of Mutations in MG. In (a) mutations with gain and loss of function are diffusely distributed in the extracellular and transmembrane areas of the receptor. In (b), the representation of these pathologic mutations is, according to the "twisted quaternary model", located among the subunits and rigid areas of the receptor. Reproduced with permission of Changeux JP 44 and the editor. 
When general anesthesia is the choice, additional care should be taken with the injection of NMB. As a rule, the reaction to muscle relaxants is unpredictable in MG patients. Regarding succinylcholine patients are often resistant to this drug requiring larger doses to obtain maximal blockade ${ }^{58}$. This can be explained by the reduced number of receptors hindering the drug from effectively depolarizing the motor plate. However, the reaction is not always one of resistance, and patients that use anticholinesterase drugs or those who underwent preoperative plasmapheresis may have a potentiating effect of succinylcholine $9,55,57,50-64$. The authors indicate that the effects are inversely proportional to the activity of plasma cholinesterase ${ }^{64}$.

Most patients seropositive or not for MG have increased sensitivity to non-depolarizing NMB ${ }^{9,56,60}$. The pattern of NMT monitoring before the injection of NMB usually is of muscle fatigue, and patients require very low doses to maintain maximum relaxation. This was seen in Case 1 (Figure 1). However, the authors observed that if before the injection of NMB the patient does not show a pattern of fatigue after evoked stimulation, the response to and the doses of NMB will be similar to those used in normal patients ${ }^{12}$. This absence of fatigue was recorded by electromyography of the pollicis adductor muscle in Case 2, in which the response to NMB followed the pattern of a normal patient (Figure 2).
Therefore, we suggest that due to the variety of response to NMB from extreme sensitivity to conventional response, similar to that observed in patients without MG, the monitoring of NMT in MG mandatory, and it should be initiated before the injection of the NMB. It has been recommended the use of a strong stimulus, such as TOF 12,65 . In addition to the information on patient response to NMB, this type of neurostimulation is equally useful in detecting residual blockade at the end of the procedure.

Recently, authors have reported successful reversion of rocuronium-induced blockade by sugammadex in patients with MG ${ }^{66,67}$. The great advantage of this new antagonist is the constancy of motor blockade reversion in MG patients, as it does not depend of interactions with preoperative anticholinesterase agents ${ }^{68}$.

To conclude, MG is defined as an autoimmune neurologic disease that affects the postsynaptic portion of the NMJ with involvement, in most cases, of the thymus. Due to the multiple forms of disease presentation, treatment, and evolution, and as a way to predict the response to NMB and its satisfactory reversion, monitoring of the NMT with TOF during anesthesia assessing muscle response before the administration of NMB is recommended. 